\title{
Reconstruction of high-resolution time series from slow-response broadband terrestrial irradiance measurements by deconvolution
}

\author{
A. Ehrlich and M. Wendisch \\ Leipzig Institute for Meteorology (LIM), University of Leipzig, Leipzig, Germany \\ Correspondence to: A. Ehrlich (a.ehrlich@uni-leipzig.de)
}

Received: 9 March 2015 - Published in Atmos. Meas. Tech. Discuss.: 21 May 2015

Revised: 13 August 2015 - Accepted: 30 August 2015 - Published: 11 September 2015

\begin{abstract}
Broadband solar and terrestrial irradiance measurements of high temporal resolution are needed to study inhomogeneous clouds or surfaces and to derive vertical profiles of heating/cooling rates at cloud top. An efficient method to enhance the temporal resolution of slow-response measurements of broadband terrestrial irradiance using pyrgeometer is introduced. It is based on the deconvolution theorem of Fourier transform to restore amplitude and phase shift of high frequent fluctuations. It is shown that the quality of reconstruction depends on the instrument noise, the pyrgeometer response time and the frequency of the oscillations.

The method is tested in laboratory measurements for synthetic time series including a boxcar function and periodic oscillations using a CGR-4 pyrgeometer with response time of $3 \mathrm{~s}$. The originally slow-response pyrgeometer data were reconstructed to higher resolution and compared to the predefined synthetic time series. The reconstruction of the time series worked up to oscillations of $0.5 \mathrm{~Hz}$ frequency and $2 \mathrm{~W} \mathrm{~m}^{-2}$ amplitude if the sampling frequency of the data acquisition is $16 \mathrm{kHz}$ or higher. For oscillations faster than $2 \mathrm{~Hz}$, the instrument noise exceeded the reduced amplitude of the oscillations in the measurements and the reconstruction failed.

The method was applied to airborne measurements of upward terrestrial irradiance from the VERDI (Vertical Distribution of Ice in Arctic Clouds) field campaign. Pyrgeometer data above open leads in sea ice and a broken cloud field were reconstructed and compared to KT19 infrared thermometer data. The reconstruction of amplitude and phase shift of the deconvoluted data improved the agreement with the KT19 data. Cloud top temperatures were improved by up to $1 \mathrm{~K}$ above broken clouds of $80-800 \mathrm{~m}$ size (1-10 s flight time) while an underestimation of $2.5 \mathrm{~W} \mathrm{~m}^{-2}$ was found for the
\end{abstract}

upward irradiance over small leads of about $600 \mathrm{~m}$ diameter ( $10 \mathrm{~s}$ flight time) when using the slow-response data. The limitations of the method with respect to instrument noise and digitalization of measurements by the data acquisition are discussed.

\section{Introduction}

Broadband solar and terrestrial irradiance are key parameters in the atmospheric energy budget. Classical instruments measuring broadband irradiance are pyranometers and pyrgeometers based on thermophile sensors (Wendisch and Brenguier, 2013).

The pyrgeometer performance is quantified by the absolute calibration accuracy, measurement stability, angular response, dome spectral transmissivity, direct solar heating and dome temperature effects (e.g., Philipona et al., 1995, 2001; Wendisch and Brenguier, 2013; Gröbner et al., 2014). For a modified Eppley Precision Infrared Radiometer (PIR), Philipona et al. (2001) quantified daytime and nighttime precision with $0.4 \%\left( \pm 1 \mathrm{~W} \mathrm{~m}^{-2}\right)$ at night and $1 \%\left( \pm 2 \mathrm{~W} \mathrm{~m}^{-2}\right)$ at day. The effect of differential temperatures between the dome and the thermophile sensor, as discussed by Albrecht and Cox (1977), was minimized by adding two additional temperature sensors in the dome (Philipona et al., 1995). For an unshaded pyrgeometer, solar radiation may cause nonhomogeneous dome temperatures in cloud-free conditions. Meloni et al. (2012) showed that this may result in an overestimation of up to $10 \mathrm{~W} \mathrm{~m}^{-2}$ for PIR pyrgeometer while for CGR-4 pyrgeometer by Kipp and Zonen this temperature effect ranges within the measurements' uncertainties. 
Gröbner et al. (2014) demonstrated that the World Infrared Standard Group (WISG) of pyrgeometer (including PIR and CGR-4) underestimates downward irradiance in clear sky conditions by 2 to $6 \mathrm{~W} \mathrm{~m}^{-2}$ compared to independent radiometers traceable to International System (IS) of Units. However, internal consistency and stability of the WISG pyrgeometers ranges within $\pm 1 \mathrm{~W} \mathrm{~m}^{-2}$.

Further uncertainties of pyrgeometer measurements result from the spectral variability of the dome transmissivity (Gröbner and Los, 2007). This mostly affects clear sky measurements with low values of integrated precipitable water vapor (IWV) causing a bias of about $-0.5 \mathrm{~W} \mathrm{~m}^{-2}$ when IWV is less than $10 \mathrm{~mm}$. However, Gröbner and Wacker (2013) showed that the spectral dome transmissivity of CGR-4 pyrgeometer is more uniform over the entire wavelength range, and IWV effects can be neglected. Similarly, effects caused by a nonuniform angular response of the pyrgeometer dome are not crucial for the overall measurement uncertainties (Albrecht et al., 1974).

One main drawback of pyranometer and pyrgeometer is their relative slow response to changes in the incident irradiance. Sensor, ambient air and dome have to be in thermal equilibrium which can take up to $6 \mathrm{~s}\left(1 \mathrm{e}^{-1}\right.$ response time) as specified for the CGR-4. While the response of the thermophile sensor itself is faster with response times of about $2 \mathrm{~s}$ (Curry and Herman, 1985). Pyranometer response times are in the same order ranging between $2 \mathrm{~s}$ for the CMP-22 by Kipp and Zonen and $15 \mathrm{~s}$ for the Eppley Standard Precision Pyranometer (SPP). For ground based measurements of the energy budget, the slow response of pyranometer and pyrgeometer is not critical. However, airborne measurements of broadband irradiance require a high temporal resolution as changes in irradiance appear more rapidly and can be faster than the response time of pyrgeometer and pyranometer. For example, vertical profiles of net broadband radiation and derived vertical heating rates depend on a dense sampling in particular near cloud top (e.g., Bucholtz et al., 2010; Duda et al., 1991; Albrecht et al., 1985). As shown by Wood (2012) the terrestrial cooling, a crucial measure influencing cloud dynamics, increases sharply within a few meters at cloud top. Therefore, analyzing the terrestrial cloud top cooling and solar heating by measurements requires a sufficient temporal resolution of pyrgeometer and pyranometer measurements.

For downward solar irradiance measured by airborne pyranometer an attitude correction is needed. If the pyranometer measurements are lagged compared to the aircraft attitude angles due to the slow response time of the pyranometer, the correction can fail. Saunders et al. (1992) introduced a time shift equal to the pyranometer response time in the irradiance time series to compensate this effect in the attitude correction. However, the reduced temporal resolution of the slowresponse pyranometer data are not corrected by adding such a time shift.

The time response of pyrgeometer measurements was already addressed by Albrecht et al. (1974) and Curry and Her- man (1985). For the PIR they investigated the consequences of different response times of the thermophile, the dome temperature and the thermophile sink. Usually the thermophile response (about $2 \mathrm{~s}$ ) is of one magnitude faster than the time the dome needs to establish equilibrium with the ambient air temperature (about $5 \mathrm{~min}$ response time). For aircraft measurements with sufficient air flow around the dome, the response time is reduced. However, Albrecht et al. (1974) as well as Curry and Herman (1985) concluded that during ascents, descents or short horizontal flight legs the pyrgeometer data are systematically biased as the stabilization of the pyrgeometer temperature equilibrium is in the order of several minutes. Foot (1986) showed that after rapid descents the equilibrium of dome and thermophile temperature of the PIR is reached after about $4 \mathrm{~min}$. Similar investigation for the CGR-4 are not available. The response time of $6 \mathrm{~s}$ specified for the CGR-4 preliminary accounts for the thermal equilibrium of sensor, dome and ambient temperature, when only the terrestrial irradiance changes. This is valid as a first approximation for flights in constant altitude (constant ambient temperature).

As a consequence previous airborne measurements focused on sampling during horizontal flight legs and integrated over several minutes to allow the sensor to establish thermodynamic equilibrium (e.g., Curry and Herman, 1985; Albrecht et al., 1985). Measurements during ascents and descents by Curry and Herman (1985) showed that the observed vertical profiles of heating rates in Arctic stratus clouds could not represent the vertical variability of heating rates derived by a radiative transfer model.

To circumvent these limitations, the spatial resolution of airborne pyrgeometer measurements can be improved by using slowly moving platforms or decreasing the ascent and descent rates in the flight pattern. In this way, Bucholtz et al. (2010) derived continuous profiles of radiative heating within a $1 \mathrm{~km}$ thick cirrus layer by extending the descent for $10 \mathrm{~min}$ with a rate of $100 \mathrm{~m} \mathrm{~min}^{-1}$. For marine stratocumulus, Duda et al. (1991) measured vertical profiles of net irradiance using a tethered balloon. This platform allows slow ascent and descent rates down to $20 \mathrm{~m} \mathrm{~min}^{-1}$. With a sampling time of about $6 \mathrm{~s}$, a vertical resolution of about $2 \mathrm{~m}$ is obtained. However, an entire profile of a $500 \mathrm{~m}$ thick cloud needs about $25 \mathrm{~min}$, which creates problems in the data interpretation. Stable horizontal homogenous conditions have to be assumed which does not hold for all cases (Duda et al., 1991).

Airborne pyrgeometer measurements of high temporal resolution are also needed to study horizontal variations of the terrestrial radiation budget. For example, above stratocumulus differences of the cloud top cooling induce vertical motion and entrainment (Wood, 2012). In the Arctic, open leads in sea ice cover may lead to enhanced sensible and latent heat fluxes and emitted terrestrial radiation (Lüpkes et al., 2008). As shown by Ruffieux et al. (1995) this effect can be observed for even small leads with a diameter down to about $100 \mathrm{~m}$. For typical aircraft velocities of at least $50 \mathrm{~m} \mathrm{~s}^{-1}$, this 
corresponds to a measurement time of only $2 \mathrm{~s}$, which is less than the response time of pyrgeometer.

To improve the temporal resolution of pyrgeometer with slow response time a method is introduced to reconstruct the fluctuations in the measured time series. The approach is based on the deconvolution theorem of Fourier transform, which is popular in different scientific fields such as the reconstruction of climate proxy time series (e.g., Bard et al., 1987).

In Sect. 2 the theory of the deconvolution theorem is described. It is exemplarily applied to pyrgeometer measurements in this paper but can also be adapted to slow-response pyranometer. The potential of the method is discussed by analyzing the minimum scales of fluctuation which can be reconstructed for different sample frequencies assuming a perfect measurement with a defined noise level. Laboratory measurements are discussed in Sect. 3 using two types of predefined time series to investigate the impact of instrument noise on the reconstruction. In Sect. 4, the reconstruction is applied to two exemplary measurement time series (leads in sea ice and broken clouds) of upward terrestrial irradiance from airborne observations. For these measurements, the limitations of the method in case of a digital data acquisition are discussed.

\section{Deconvolution of time series}

\subsection{Theory}

An instantaneous change of a given quantity can be described with the Heaviside step function $\mathcal{H}(t)$, defined by $\mathcal{H}(t \leq 0)=X_{0}$ and $\mathcal{H}(t>0)=X_{0}+\Delta X$. In that case, the response of pyrgeometer and pyranometer follows an exponential decay with time $t$ characterized by the response time $\tau$. The temporal instrument readout $X(t)$ as a response of the instantaneous change of the measured quantity is described by

$X(t)=X_{0}+\Delta X \cdot \exp \left(-\frac{t}{\tau}\right)$,

with the response time $\tau$ defining the time when the difference between readout and actual value reaches $1 / e(37 \%)$ of the initial difference $\Delta X$. The function given by Eq. (1) represents a convolution $(\mathcal{H} * g)(t)$ of the step function $\mathcal{H}(t)$ with the convolution kernel $g(t)$,

$X(t)=(\mathcal{H} * g)(t)=\int_{-\infty}^{\infty} \mathcal{H}\left(t^{\prime}\right) \cdot g\left(t-t^{\prime}\right) \mathrm{d} t^{\prime}$.

For an arbitrary change of the measured quantity $x(t)$ the instrument readout is similarly described by

$X(t)=(x * g)(t)=\int_{-\infty}^{\infty} x\left(t^{\prime}\right) \cdot g\left(t-t^{\prime}\right) \mathrm{d} t^{\prime}$.
In case of an exponential response characteristic of the instrument it holds:

$g(t)= \begin{cases}1 / \tau \cdot \exp (t / \tau), & t \leq 0 \\ 0, & t>0 .\end{cases}$

Freese and Kottmeier (1998) applied an approximation to correct for the slow time response of pyranometer and pyrgeometer by using the Newton's formula including the first derivative of the measurement time series by,

$x(t)=X(t)+\tau \cdot \frac{\mathrm{d} X(t)}{\mathrm{d} t}$.

However, an exact analytical solution for the convolution is given by the convolution theorem of Fourier transform:

$\mathcal{F}\{X\}=\mathcal{F}\{x * g\}=\mathcal{F}\{x\} \cdot \mathcal{F}\{g\}$,

with $\mathcal{F}\{X\}, \mathcal{F}\{x\}$, and $\mathcal{F}\{g\}$ the Fourier transform of the instrument readout, the actual measured time series and the convolution kernel, respectively. If the convolution kernel $g$ is known, Eq. (6) can easily be inverted and used to calculate the Fourier transform of the actual time series not smoothed by the delayed instrument response,

$\mathcal{F}\{x\}=\frac{\mathcal{F}\{X\}}{\mathcal{F}\{g\}}$.

By inverse Fourier transformation of $\mathcal{F}\{x\}$ the original time series $x(t)$ can be derived,

$x(t)=\int_{-\infty}^{\infty} \mathcal{F}\{x\} \cdot e^{i \cdot 2 \pi f \cdot t} \mathrm{~d} f$.

In practice, the reconstruction may fail when instrument noise is artificially amplified. To suppress these effects, an additional low pass filter (moving average) is applied and the inverse Fourier transformation calculated only from frequencies below a specified cut-off frequency $f_{\mathrm{c}}$. The Fourier transform of a moving average (boxcar function) with window length of $T_{\mathrm{m}}$ in units of seconds is given by the sinc function what finally results in the inverse transformation,

$x(t)=\int_{-f_{\mathrm{c}}}^{f_{\mathrm{c}}} \operatorname{sinc}\left(T_{\mathrm{m}} \cdot f\right) \cdot \mathcal{F}\{x\} \cdot e^{i \cdot 2 \pi f \cdot t} \mathrm{~d} f$.

The deconvolution of long time series of high sampling frequency is demanding. Fortunately, due to the exponential kernel function a single measurement of a time series is affected only by measurements within a certain time span. The fast decay of the exponential kernel function is used to accelerate the computational speed by splitting the time series into smaller sections. A criteria of $99.99 \%$ decay and corresponding time segments of $t_{\text {split }}=\tau \cdot \ln \left(10^{4} \cdot \tau^{-1}\right)$ should be sufficient. A section length of $(10+2) \cdot t_{\text {split }}$ including an overlap 
of overlap $2 \cdot t_{\text {split }}$ to glue the single sections, was chosen for further investigations in this paper. For example, in case of a response time of $\tau=4 \mathrm{~s}$ the section length is about $6 \mathrm{~min}$, the overlap $1 \mathrm{~min}$. The overlap is used for the computation of the deconvolution only and rejected when gluing the section into the final entire time series.

\subsection{Reconstruction of synthetic time series}

To demonstrate the potential of the deconvolution method to reconstruct pyrgeometer measurements synthetic time series of convoluted data are analyzed. The convoluted time series represent ideal measurements without any noise. In this way, it is studied how large the amplitude of fluctuations is in the measured time series and if this damped amplitudes range above or below the noise level of the instrument. For the original time series a periodic function with symmetric rise and decline was chosen. The frequency of the oscillations $f_{\mathrm{p}}$ was varied between 0.1 and $4 \mathrm{~Hz}$. The corresponding theoretically measured time series were calculated by convolution taking into account the response time $\tau$ in the range of 0.1$10 \mathrm{~s}$. The convoluted time series were calculated for a sampling frequency of $f_{\mathrm{s}}=20 \mathrm{~Hz}$, which is typical for airborne pyrgeometer and pyranometer measurements.

In Fig. 1 three exemplary time series with frequencies $f_{\mathrm{p}}=0.25 / 1 / 2 \mathrm{~Hz}$ are analyzed. A hypothetical noise level of $0.25 \mathrm{~W} \mathrm{~m}^{-2}$ is illustrated by the gray area (typical for CGR-4 pyrgeometer used in this paper). The original time series (red line) with amplitude $\pm 5 \mathrm{~W} \mathrm{~m}^{-2}$ is convoluted for different instrument response times. In all cases, a decrease of measured amplitude and an increasing of the phase shift with increasing response time is obvious. With increasing frequency of the fluctuations the phase shift and the amplitude of the measured time series become smaller. For $\tau=4 \mathrm{~s}$ the maximum amplitude is $1.8 \mathrm{~W} \mathrm{~m}^{-2}$ for $f_{\mathrm{p}}=0.25 \mathrm{~Hz}$, while for $f_{\mathrm{p}}=2 \mathrm{~Hz}$ the maximum amplitude of $0.25 \mathrm{~W} \mathrm{~m}^{-2}$ is close to the noise level of pyrgeometer measurements. In this case, the small damped fluctuations registered in the measurements might be superimposed by noise and the reconstruction of the given fluctuations by deconvolution is not meaningful.

A signal to noise ratio of $\Delta F_{\mathrm{c}} / \Delta F_{\mathrm{n}} \geq 1$ between the amplitude of the convoluted data $\Delta F_{\mathrm{c}}$ and the noise amplitude $\Delta F_{\mathrm{n}}$ was defined as a criteria of the ability to reconstruct a specific time series. We assumed a noise amplitude of $\Delta F_{\mathrm{n}}=0.25 \mathrm{~W} \mathrm{~m}^{-2}$ and translated the results of the calculations into a specific minimum amplitude $\Delta F_{\min }$ representing the smallest periodic fluctuations which from theory can be reconstructed from the measurements. Taking into account the amplitude of the original time series of $\Delta F=10 \mathrm{~W} \mathrm{~m}^{-2}$ it is

$$
\Delta F_{\min }=\frac{\Delta F}{\Delta F_{\mathrm{c}}} \cdot \Delta F_{\mathrm{n}}
$$

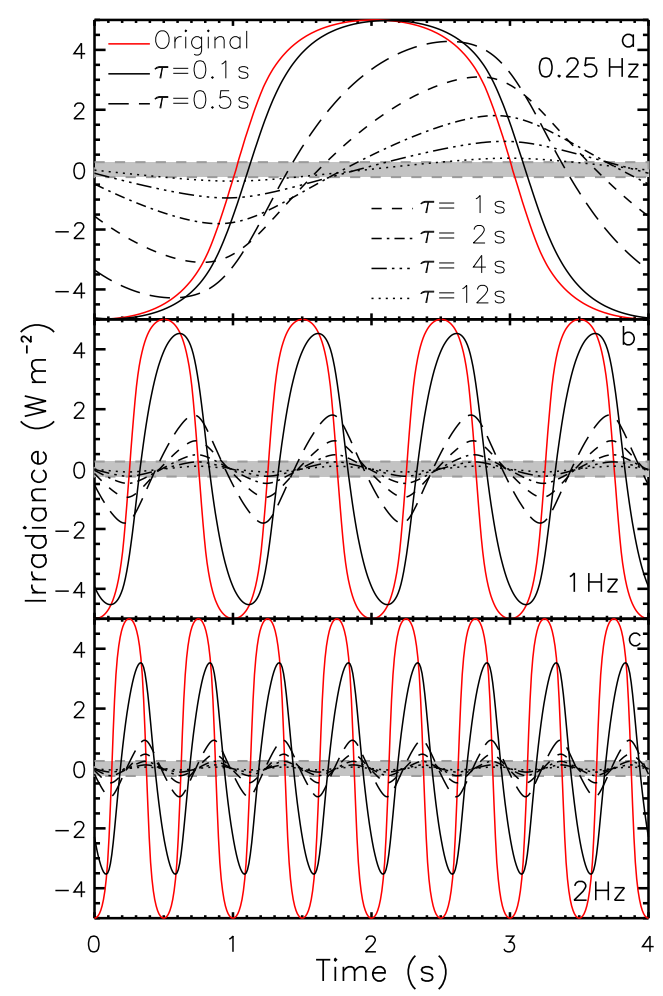

Figure 1. Original synthetic time series (red) and convoluted time series (black) of irradiance for three different frequencies $f_{\mathrm{p}}=$ $0.25 / 1 / 2 \mathrm{~Hz}(\mathbf{a}-\mathbf{c})$. The different black lines show convoluted time series for different response times $\tau$ between 0.1 and $12 \mathrm{~s}$. The gray area indicates a hypothetical noise level of $0.25 \mathrm{~W} \mathrm{~m}^{-2}$.

These minimum amplitudes are presented in Fig. 2a as function of response time $\tau$ of the sensor and frequency of the fluctuations $f_{\mathrm{p}}$ showing an increase of $\Delta F_{\min }$ with increasing $\tau$ and $f_{\mathrm{p}}$. For slow response times and slow oscillations, the fluctuations with amplitudes below $0.5 \mathrm{~W} \mathrm{~m}^{-2}$ can still be resolved while $\Delta F_{\min }$ can exceed $10 \mathrm{~W} \mathrm{~m}^{-2}$ for frequencies above $1 \mathrm{~Hz}$ and response times larger than $2 \mathrm{~s}$. Figure 2a suggests a linear dependence between $\Delta F_{\min }$ and the product $\left(\tau \cdot f_{\mathrm{p}}\right)$. The product of $\tau$ and $f_{\mathrm{p}}$ can be interpreted as ratio of the characteristic times of sensor $(\tau)$ and oscillations $\left(1 / f_{\mathrm{p}}\right)$ as it is shown in Fig. 2b. For low values of $\left(\tau \cdot f_{\mathrm{p}}\right)$, the minimum amplitudes are close to the predefined noise criteria what means that almost all fluctuations above the noise criteria can be reconstructed. For $\left(\tau \cdot f_{\mathrm{p}}\right)>0.5$ the plot follows an almost perfect linear behavior given by

$\Delta F_{\min }=1.3 \cdot\left(\tau \cdot f_{\mathrm{p}}\right)$.

All fluctuations above this line can potentially be reconstructed.

In general, this linear relation depends on the defined noise amplitude $\Delta F_{\mathrm{n}}$. The lower the noise of the instrument, the lower the amplitudes that can be resolved by deconvolution. This relation is linear meaning that reducing the noise level 

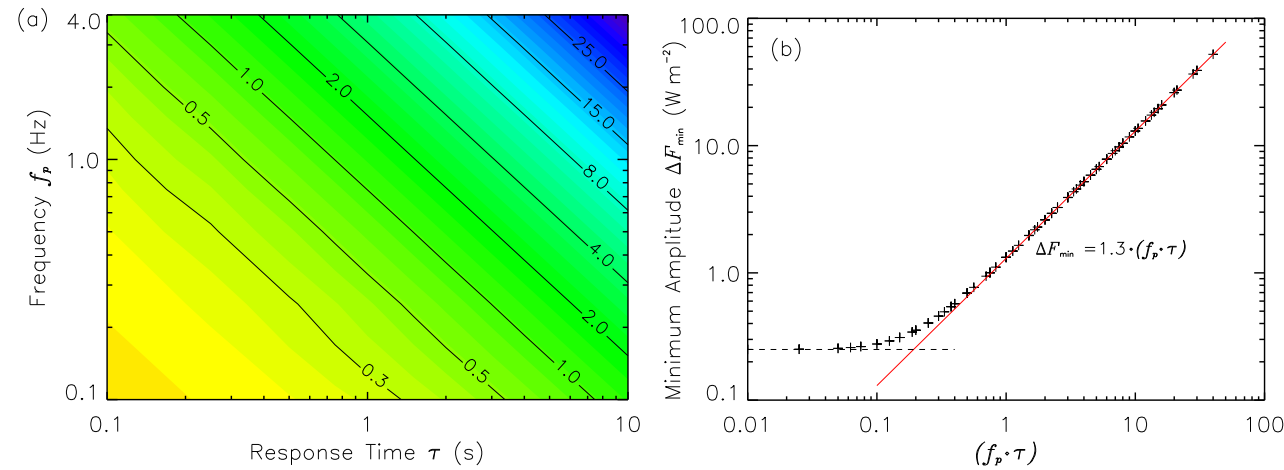

Figure 2. (a) Minimum amplitude $\Delta F_{\text {min }}$ of fluctuations which theoretically can be resolved by deconvolution in dependence of the sensor response time $\tau$ and the oscillation frequency $f_{\mathrm{p}}$. (b) Similar to (a) $\Delta F_{\min }$ is given as a function of the product $\left(f_{\mathrm{p}} \cdot \tau\right)$. Symbols illustrate the results of the calculation. The red line indicates a linear fit for large $\left(f_{\mathrm{p}} \cdot \tau\right)$.

by a factor of 2 reduces the minimum amplitudes by a factor of 2. Based on the fit given in Eq. (11) (Fig. 2b) the following relation is postulated:

$\Delta F_{\min }=A \cdot \Delta F_{\mathrm{n}} \cdot\left(\tau \cdot f_{\mathrm{p}}\right)$.

The empirical parameter $A$ depends on the shape of the original periodic time series. For the shape of the oscillations used here $A=5.2$ was determined. Calculations, not shown here, using a square wave (boxcar) function give a smaller coefficient with $A=4$ while for a triangular wave function $A$ is larger with $A=8$. This illustrates that the theoretical capability of deconvolution increases for sharper fluctuations. For fluctuations with a continuous increase and decrease (triangular wave) $\Delta F_{\min }$ is lower by a factor of 2 compared to an instantaneous increase and decrease of the oscillations (square wave). Other shapes of fluctuations will likely vary between these two extremes. For a periodic but not symmetric oscillation based on the laboratory measurements presented in Sect. 3, $A=6.4$ was determined, which is slightly closer to the triangle function than the more smooth oscillation presented here.

\section{Laboratory measurements}

\subsection{Instrumentation}

A CGR-4 pyrgeometer manufactured by Kipp and Zonen was used in combination with the analog signal amplifier CT 24 also provided by Kipp and Zonen. The amplified thermoelectric voltage was digitized by an analog-to-digital (AD) converter USB-6009 by National Instruments. The AD converter sampled with up to $48 \mathrm{kHz}$ sampling frequency and 14 bit resolution. Three AD channels were utilized, one for the pyrgeometer voltage and two for the thermistor (input and output voltage) measuring the internal sensor temperature inside the CGR-4. This reduced the sampling frequency $f_{\mathrm{s}}$ to $16 \mathrm{kHz}$. To investigate the net terrestrial irradiance two pyrgeometer (upward and downward facing) are needed. In the case of two CGR-4 being logged with the same AD converter, the sampling frequency is reduced to $f_{\mathrm{s}}=8 \mathrm{kHz}$. If additionally solar pyrgeometer data are logged, a maximum sampling frequency of $f_{\mathrm{s}}=4 \mathrm{kHz}$ can be obtained for such kind of $\mathrm{AD}$ converter.

The full time series sampled at $48 \mathrm{kHz}$ can not be saved. Therefore, a recording frequency of $20 \mathrm{~Hz}$ was selected. All samples in between are averaged reducing the noise in the measurements significantly.

As discussed by Albrecht et al. (1974) the pyrgeometer thermophile and the dome temperature exhibit different response times. Therefore, in this section only net irradiance measured by the pyrgeometer thermophile were analyzed, which excludes additional effects due to the time response of the internal thermistor. For the sake of comparison, all irradiance time series were subtracted by the mean irradiance measured by the pyrgeometer highlighting the amplitude of the fluctuations in the time series.

\subsection{Boxcar function}

Irradiance time series with shape of a boxcar function were generated to determine the response time of the pyrgeometer. The pyrgeometer dome was covered for $40 \mathrm{~s}$ by a plate with higher temperature compared to the ambient air. The data were recorded with $f_{\mathrm{r}}=20 \mathrm{~Hz}$ and different sampling frequencies of $f_{\mathrm{s}}=16 / 8 / 4 \mathrm{kHz}$. Examples of the measured time series are given in Fig. 3. Within the $40 \mathrm{~s}$, the measured irradiance (black line) approaches the emitted irradiance by the plate (gray line). After the plate was removed the temporal decay of the measured irradiance was analyzed as an alternative measure of the response time. For both increase and decrease $\tau$ was calculated, estimating the time of $63 \%$ increase or decay. For the CGR-4 used here, a mean value from increase and decrease of $\tau=3.3 \mathrm{~s}$ was calculated and used to reconstruct the original boxcar function. 

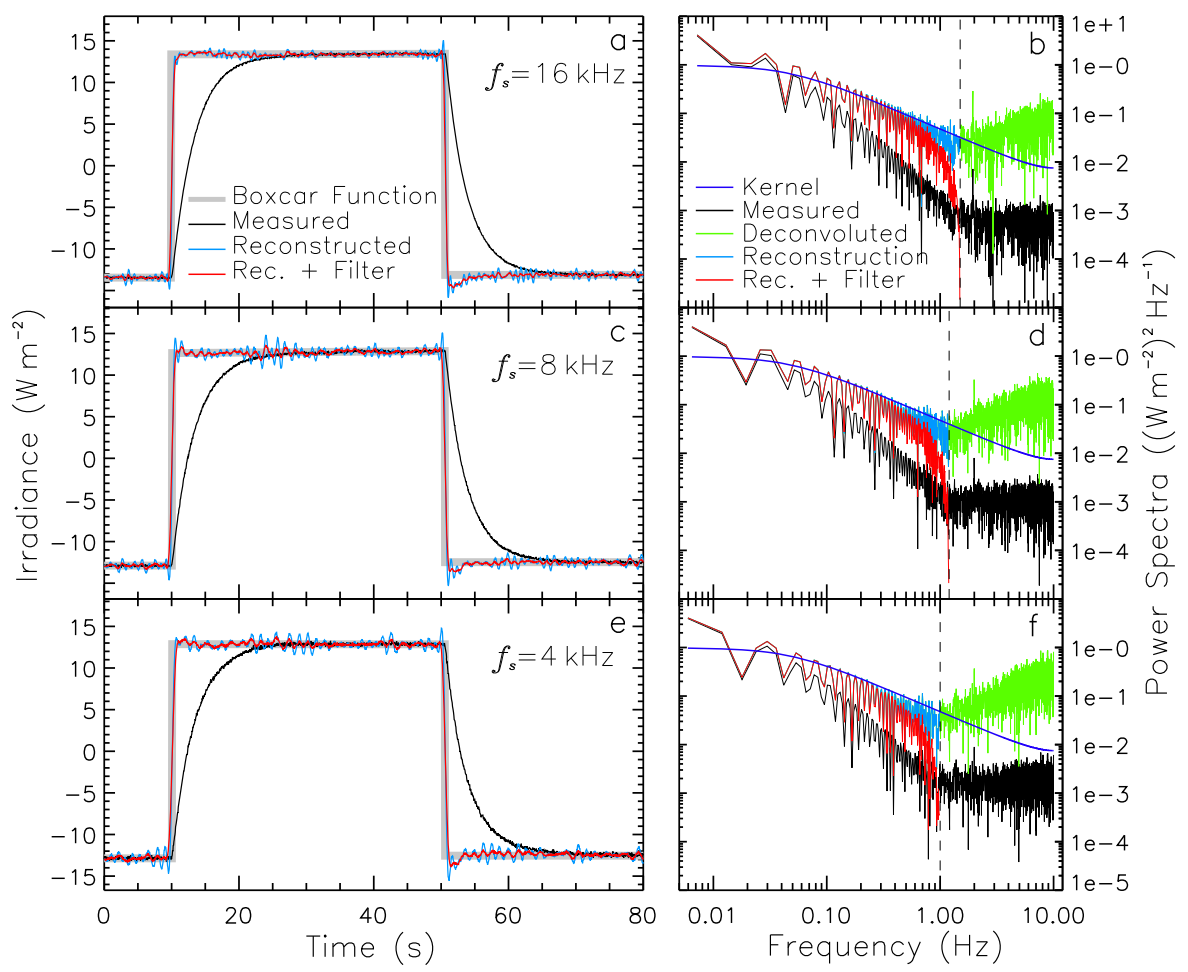

Figure 3. Measured (black) and reconstructed irradiance without filter (blue) and with filter (red) for an original boxcar function (gray) for three different sampling frequencies $f_{\mathrm{s}}=16 / 8 / 4 \mathrm{kHz}$ (a, $\mathbf{c}$ and $\mathbf{d}$ ). The corresponding power spectra for the measured (black), deconvoluted (green) and reconstructed time series (blue and red) as well as the convolution kernel (dark blue) are given in (b, $\mathbf{d}$, and f).

The time series was reconstructed for all three measurements with different sampling frequencies (compare Fig. 3a, c and e). The corresponding power spectra of measured and deconvoluted time series are given in the right panels of Fig. 3. Additionally, the Fourier transform of the kernel (exponential decay, Eq. 4) and the frequencies used for the inverse transformation are illustrated. The low-pass filter characteristic of the kernel (slow sensor response) is obvious in the decay of the kernel Fourier transform for high frequencies. The convolution damps the Fourier coefficients, and thus the amplitudes of fluctuations with high frequencies. However, the scale break of the decay at high frequencies in the power spectra of the measurements shows that the data are contaminated by instrument noise. The noise significantly differs for the three measurement examples as the scale break is shifted to smaller frequencies for lower sampling frequency $f_{\mathrm{s}}$.

By deconvolution as given in Eq. (7) these frequencies are artificially amplified as indicated by the increasing power spectra of the deconvoluted time series for high frequencies. To suppress this noise in the reconstructed time series different cut-off frequencies $f_{\mathrm{c}}$ were applied when calculating the inverse transformation using Eq. (9). For a sampling frequency of $f_{\mathrm{s}}=16 \mathrm{kHz}$ the noise of the measurements is lowest with an amplitude of $\Delta F_{\mathrm{n}}=0.08 \mathrm{~W} \mathrm{~m}^{-2}$, which allows for the use a high cut-off frequency of $f_{\mathrm{c}}=1.5 \mathrm{~Hz}$. With a re- duced sampling frequency $\left(f_{\mathrm{s}}=8 / 4 \mathrm{kHz}\right)$ the noise amplitude of the measurements increases $\left(0.12 / 0.25 \mathrm{~W} \mathrm{~m}^{-2}\right)$ and lower cut-off frequencies were feasible $\left(f_{\mathrm{c}}=1.2 / 1.0 \mathrm{~Hz}\right)$.

The general structure of the original boxcar function could be reconstructed in all three examples, indicating that the response time was correctly determined. The steps in the boxcar function were reproduced with the accuracy expected from the chosen cut-off frequency. A closer look into the data showed that the step takes about $0.65 \mathrm{~s}$ in the reconstructed time series for $f_{\mathrm{s}}=16 \mathrm{kHz}$, which is close to the inverse of the cut-off frequency of $f_{\mathrm{c}}=1.5 \mathrm{~Hz}$. Additionally, no differences for the positive and negative step of the boxcar function are found, which is in agreement with the symmetry of the pyrgeometer response discussed by Wilson et al. (2009).

In all cases small oscillations are present in the reconstructed data. This still might be a result of the instrument noise although the cut-off frequencies were chosen carefully to reduce noise. Using higher $f_{\mathrm{c}}$, increased the oscillations, but for lower $f_{\mathrm{c}}$ the oscillations could not be removed completely. This indicates that the remaining oscillations result primarily from the Gibbs phenomenon. At a jump discontinuity as represented by the boxcar function, the application of the Fourier transform is limited, causing ringing artifacts in the inverse transformation if the number of coefficients in the Fourier transform is not infinite. Therefore, the boxcar function is an extreme to illustrate how the deconvolution 
acts for different instrument noise levels. In real pyrgeometer measurements sharp changes are not typical.

The oscillations can be reduced by applying an additional moving average filter as described in Eq. (9) which acts similar to the sigma-approximation often used to eliminate the Gibbs phenomenon. Applying filter with window length $T_{\mathrm{m}}=1 / f_{\mathrm{c}}$ did significantly improve the results shown in Fig. 3 (red lines). The magnitude of the oscillations is reduced but consequently, the width of the step slightly increases to about $1 \mathrm{~s}$ what is still in the range of the inverse of the cut-off frequency. As this might be different in other cases, the use of a moving average filter has always to be weighted in each individual case with the negative consequence of a reduced temporal resolution caused by the smoothing. Similar to the results without filter, the remaining oscillations again become larger when the sensor noise is increased due to a reduction of the sampling frequency.

\subsection{Periodic function}

Continuous oscillating time series of terrestrial irradiance were generated using a chopper wheel. The chopper was operated in front of the pyrgeometer sensor head and rotated with frequencies $f_{\mathrm{p}}$ in the range between 0.1 and $2 \mathrm{~Hz}$. Behind the chopper a plate with a temperature higher than the chopper wheel was placed. The exact irradiance time series generated by the chopper was determined by measurements at fixed chopper positions. For this case, (fixed chopper) each measurement was taken long after the slow-response pyrgeometer showed stable values (after about $20 \mathrm{~s}$ ). During the measurements the temperatures of plate and chopper wheel were additionally observed by an infrared thermometer. Changing the temperature of the plate allowed for the study of the response of the pyrgeometer to periodic functions with different amplitudes between 1 and $8 \mathrm{~W} \mathrm{~m}^{-2}$.

\subsubsection{Influence of sampling frequency $f_{\mathrm{s}}$}

To illustrate the influence of different sampling frequencies (similar to Sect. 3.2), first measurements of slow oscillations $f_{\mathrm{p}}=0.1 \mathrm{~Hz}$ were investigated. Three sampling frequencies, $f_{\mathrm{s}}=16 / 8 / 4 \mathrm{kHz}$, were applied. For all sampling frequencies $20 \mathrm{~s}$ time periods of measured and reconstructed terrestrial irradiance are shown in Fig. 4. The mean value of the period was subtracted in order to provide time series oscillating around zero. Two different amplitudes of oscillations were illustrated representative for high $\left(\Delta F=8 \mathrm{~W} \mathrm{~m}^{-2}\right.$, left panels) and low ( $\Delta F=2 \mathrm{~W} \mathrm{~m}^{-2}$, center panels) fluctuations. The corresponding Fourier power spectra are shown in the right panels.

Depending on the sampling frequency, the recording produced different noise levels which are obvious in the originally measured irradiance (black lines) and in the flattening of the power spectra, which are shifted to lower frequencies for low sampling frequencies. From the $16 \mathrm{kHz}$ data the noise amplitude of the measurements was about $\Delta F_{\mathrm{n}}=0.08 \mathrm{~W} \mathrm{~m}^{-2}$. For 8 and $4 \mathrm{kHz}$ higher noise levels with $\Delta F_{\mathrm{n}}=0.12 \mathrm{~W} \mathrm{~m}^{-2}$ and $\Delta F_{\mathrm{n}}=0.25 \mathrm{~W} \mathrm{~m}^{-2}$ were found. From Eq. (12) (with $A=6.4$ ) minimum amplitudes of $\Delta F_{\min }=0.5 \mathrm{~W} \mathrm{~m}^{-2}$ for the noise level are derived indicating that reconstruction works in all cases investigated here using the full deconvoluted spectra.

In practice a cut-off frequency of $f_{\mathrm{c}}=1 \mathrm{~Hz}$ was chosen for the reconstruction, which is sufficient to reduce noise and adequately recover $0.1 \mathrm{~Hz}$ oscillations. In this case - a relativly slow oscillation compared to the response time and sampling frequency - an additional low pass filter (moving average) with window length $T_{\mathrm{m}}=1 \mathrm{~s}$ could be applied to further reduce the impact of noise on the deconvolution. The chosen window length is short enough to avoid a reduction of the amplitude of the reconstructed data. The reduction of noise is shown in the decrease of the power spectra used for the reconstruction (red lines) close to the cut-off frequency. These power spectra are significantly lower than the power spectra obtained after the deconvolution (green lines).

The comparison of reconstructed and original irradiance measurements showed in general differences below $0.5 \mathrm{~W} \mathrm{~m}^{-2}$ for both low and high amplitude and all sampling frequencies. The general shape of the time series generated by the chopper was reconstructed by deconvolution and the phase shift was removed. However, small scale fluctuations are obvious in the reconstructions of the low amplitude oscillations using measurements with $f_{\mathrm{s}}=8 \mathrm{kHz}$ and $f_{\mathrm{s}}=4 \mathrm{kHz}$. This illustrates that even when applying a conservative cutoff frequency and an additional low pass filter high sampling frequencies (low noise) improve the quality of deconvolution.

The differences between original and reconstructed irradiance were quantified by their normalized percentaged standard deviation (SD) $c_{\mathrm{V}}=\sigma / \Delta F \cdot 100 \%$. The results given in Table 1 show that for the oscillation with high amplitude $\Delta F=8 \mathrm{~W} \mathrm{~m}^{-2}$ the differences are almost equal for all sampling frequencies $f_{\mathrm{s}}=16 / 8 / 4 \mathrm{kHz}$ with $c_{\mathrm{V}}$ ranging between 2 and $3 \%$. For the chopping with lower amplitude $\Delta F=2 \mathrm{~W} \mathrm{~m}^{-2}$ the relative differences are higher ranging between 4 and $8 \%$. A tendency of higher $c_{\mathrm{V}}$ for lower $f_{\mathrm{s}}$ is found. Both is closely related to the signal to noise ratio, which is lower for the lower amplitudes, and decreases with sampling frequency. Not observing this pattern for the oscillations with high amplitude indicated that here the sampling frequency is sufficient in all cases.

\subsubsection{Influence of oscillation frequency $f_{\mathrm{p}}$}

Figure 5 shows reconstructed time series for different oscillations with $f_{\mathrm{p}}=0.2 / 0.5 / 2 \mathrm{~Hz}$. For the sake of comparison, a low pass filter was not applied because the filter would cause a reduction of the oscillation amplitudes if the window length is chosen in a similar range as in Sect. 3.3.1. For $f_{\mathrm{p}}=0.2 \mathrm{~Hz}$ and $f_{\mathrm{p}}=0.5 \mathrm{~Hz}$, a cut-off frequency of $1 \mathrm{~Hz}$ was chosen for 


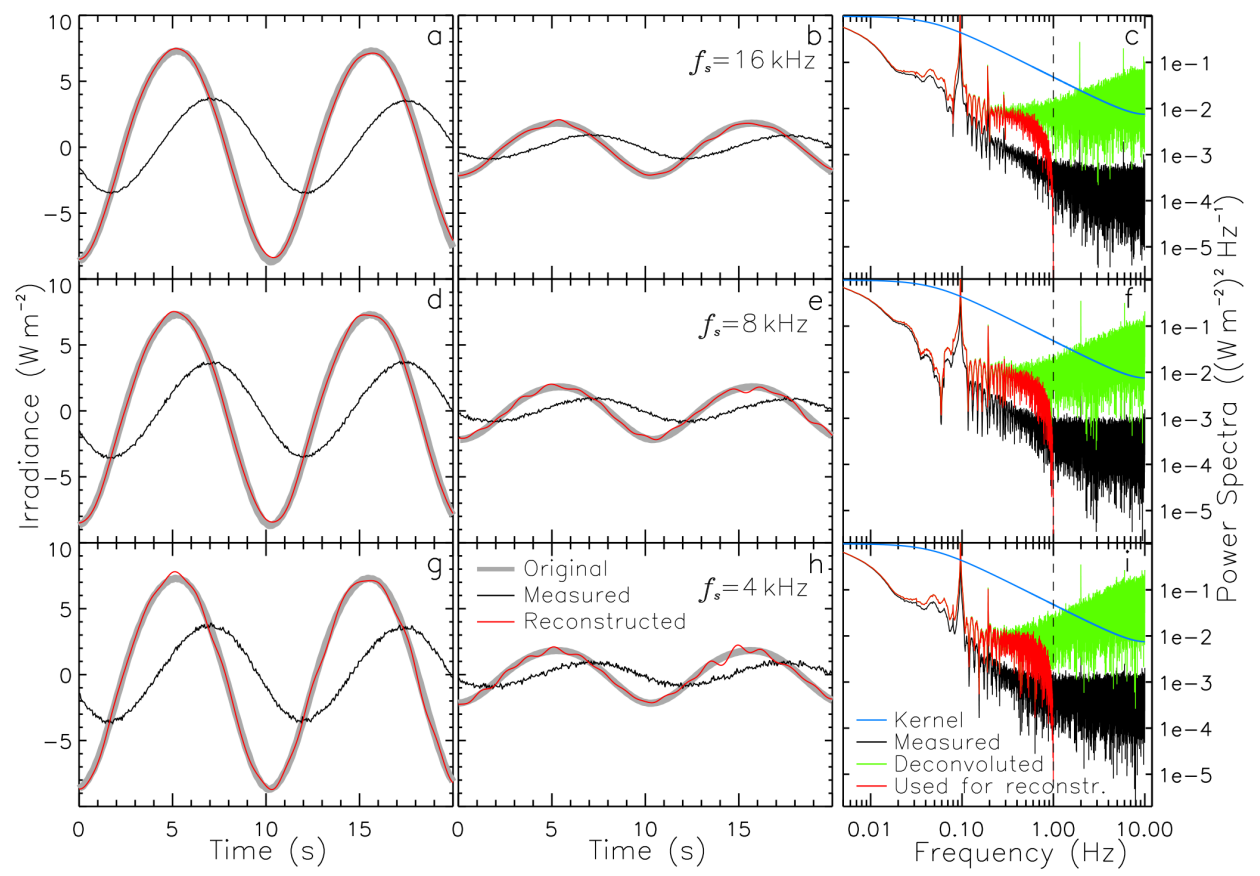

Figure 4. Measured (black) and reconstructed irradiance (red) in case of a periodic oscillating time series (gray) for three different sampling frequencies $f_{\mathrm{s}}=16 / 8 / 4 \mathrm{kHz}$. Oscillations with two different amplitude were generated, $8 \mathrm{~W} \mathrm{~m}^{-2}$ amplitude in the left panels (a, $\mathbf{d}$ and $\mathbf{g}$ ) and $2 \mathrm{~W} \mathrm{~m}^{-2}$ amplitude in the center (b, e and $\mathbf{h}$ ). The corresponding power spectra for the measured (black), deconvoluted (green) and reconstructed time series (red) as well as the convolution kernel (blue) are given in (c, $\mathbf{f}$ and $\mathbf{i})$.

the reconstruction, while for $f_{\mathrm{p}}=2 \mathrm{~Hz}$ a cut-off frequencies of $4 \mathrm{~Hz}$, higher than the oscillation frequency, was applied.

Applying Eq. (12) to calculate the potential of deconvolution for these oscillations $(A=6.4)$ gives $\Delta F_{\min }=$ $0.34 \mathrm{~W} \mathrm{~m}^{-2}$ for $f_{\mathrm{p}}=0.2 \mathrm{~Hz}$ and $\Delta F_{\min }=0.85 \mathrm{~W} \mathrm{~m}^{-2}$ for $f_{\mathrm{p}}=0.5 \mathrm{~Hz}$. Both minimum amplitudes range below the actual amplitude of the oscillating irradiance time series. However, for $f_{\mathrm{p}}=2 \mathrm{~Hz}$ a minimum amplitude of $3.4 \mathrm{~W} \mathrm{~m}^{-2}$ is derived for which at least the oscillation with low amplitude $\Delta F=2 \mathrm{~W} \mathrm{~m}^{-2}$ should disappear in the sensor noise.

Figure 5 shows that for $f_{\mathrm{p}}=0.2 \mathrm{~Hz}$ and $f_{\mathrm{p}}=0.5 \mathrm{~Hz}$ the reconstructed and theoretical irradiance again agree. For the oscillations with high amplitude (Fig. 5a and d), the corresponding normalized percentaged SD given in Table 1 are in the range observed for the slow oscillations of Sect. 3.3.1 with $c_{\mathrm{v}}=3.4 \%$ and $c_{\mathrm{v}}=7.2 \%$. For the oscillations with low amplitude (Fig. 5b and e) the deviations are larger and apparent in a ripple structure of the reconstructed irradiance. Here $c_{\mathrm{v}}$ increased up to $10 \%$, which indicates that the signal to noise ratio is lower in these cases and moderately affects the accuracy of the reconstruction. Especially for $f_{\mathrm{p}}=0.5 \mathrm{~Hz}$ the amplification by deconvolution of high frequencies in the power spectra affects also frequencies used for the reconstruction.

Figure $5 \mathrm{~g}, \mathrm{~h}$ and i show the results for an oscillation with $f_{\mathrm{p}}=2.0 \mathrm{~Hz}$. For both low and high amplitudes, the reconstruction by deconvolution fails. Although the oscillation is
Table 1. Normalized percentaged SD $c_{\mathrm{V}}$ between original and reconstructed irradiance for measurements of different oscillations, as illustrated in Figs. 4 and 5.

\begin{tabular}{|c|c|c|c|}
\hline$f_{\mathrm{p}}(\mathrm{Hz})$ & $f_{\mathrm{s}}(\mathrm{kHz})$ & $\begin{array}{r}c_{\mathrm{V}}(\%) \\
\Delta F=8 \mathrm{~W} \mathrm{~m}^{-2}\end{array}$ & $\begin{array}{r}c_{\mathrm{V}}(\%) \\
\Delta F=2 \mathrm{~W} \mathrm{~m}^{-2}\end{array}$ \\
\hline 0.1 & 16 & 2.5 & 4.0 \\
\hline 0.1 & 8 & 2.0 & 6.9 \\
\hline 0.1 & 4 & 2.8 & 8.3 \\
\hline 0.2 & 16 & 3.4 & 9.1 \\
\hline 0.5 & 16 & 7.2 & 10.2 \\
\hline 2.0 & 16 & 29.3 & 63.8 \\
\hline
\end{tabular}

in phase and the amplitude is partly correct, in this case the results of the reconstruction are not reliable. A $c_{\mathrm{V}}$ of 29 and $64 \%$ is given in Table 1 . The main reason for these differences is the use of a higher cut-off frequency of $4 \mathrm{~Hz}$ to cover the fast oscillations. This did increase the influence of noise as obvious in the power spectra. Here the frequency range above $1 \mathrm{~Hz}$ where the power spectra increases with frequency, indicating an amplification of noise, dominates the reconstructed time series. This illustrates that such oscillations of high frequencies can not be reconstructed with the instrument setup used here. 


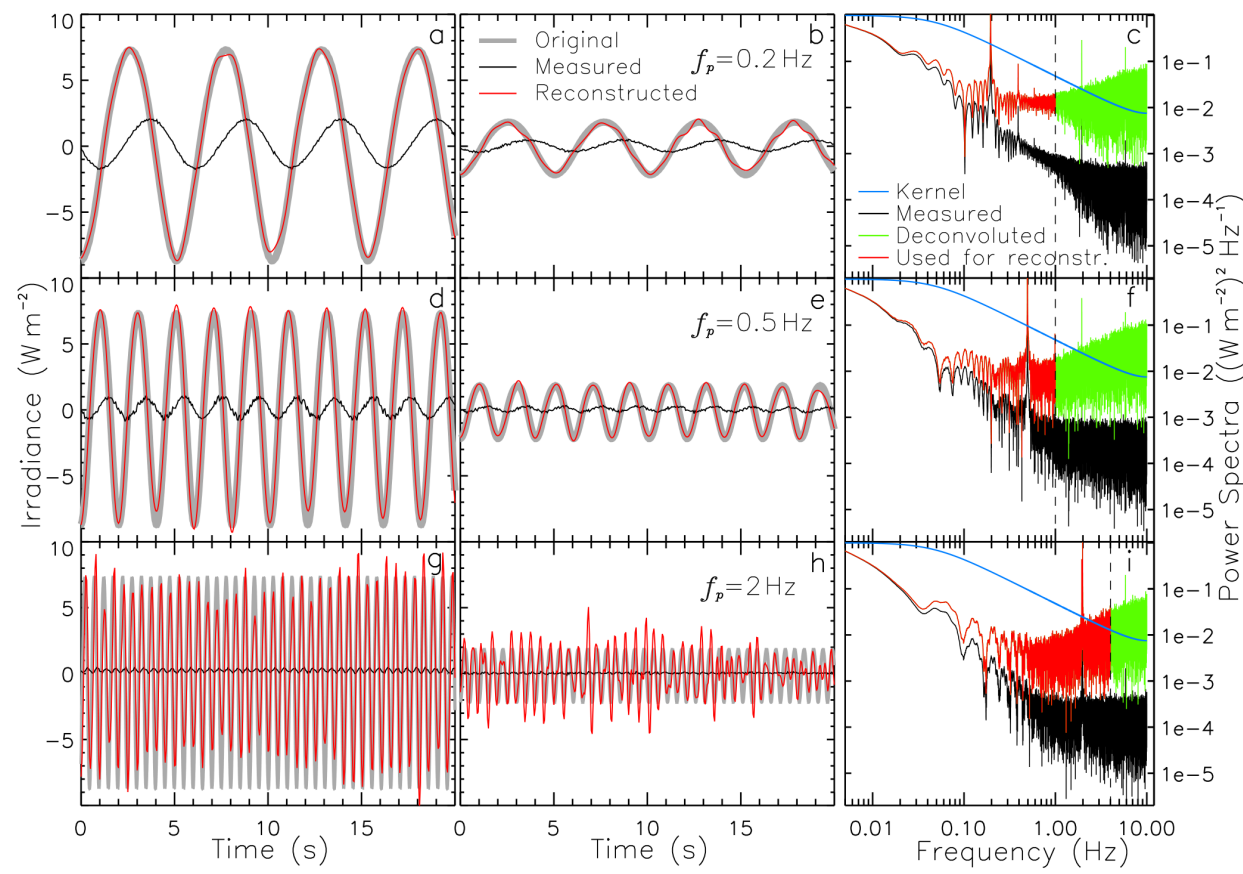

Figure 5. Measured (black) and reconstructed irradiance (red) in case of a periodic oscillating time series (gray) for three different frequencies of the oscillation $f_{\mathrm{p}}=0.2 / 0.5 / 2 \mathrm{~Hz}$. Oscillations with two different amplitude were generated, $8 \mathrm{~W} \mathrm{~m}^{-2}$ amplitude in the left panels (a, $\mathbf{d}$ and $\mathbf{g}$ ) and $2 \mathrm{~W} \mathrm{~m}^{-2}$ amplitude in the center panels (b, e and $\mathbf{h}$ ). The corresponding power spectra for the measured (black), deconvoluted (green) and reconstructed time series (red) as well as the convolution kernel (blue) are given in (c, f, and i).

\section{Application}

Airborne measurements of broadband terrestrial radiation from the international field campaign Vertical Distribution of Ice in Arctic Clouds (VERDI) were used to demonstrate the application of the deconvolution method. VERDI was based in Inuvik, Northwest Territories, Canada, and took place in April and May 2012. It included 13 research flights over the Canadian Beaufort Sea. The research aircraft Polar 5 of the Alfred Wegener Institute for Polar and Marine Research (AWI) was equipped with remote sensing and in situ instruments with the purpose to investigate Arctic clouds (Schäfer et al., 2015; Klingebiel et al., 2015). In this paper, upward terrestrial irradiance measured by a Kipp and Zonen CGR-4 pyrgeometer and upward nadir brightness temperatures from a Heitronics KT19.85 II are analyzed. Both instruments were operated with $20 \mathrm{~Hz}$ sampling frequency. The CGR-4 was amplified with the digital signal amplifier AMPBOX by Kipp and Zonen providing a digital resolution of about $0.2 \mathrm{~W} \mathrm{~m}^{-2}$. For noise reduction, the AMPBOX applies a low pass filter averaging the signal over $440 \mathrm{~ms}$. The response time of the KT19 is specified by an internal bessel filter of second order with $\tau=0.43 \mathrm{~s}$ while for the CGR-4 the response time $\tau=3.3 \mathrm{~s}$ of the laboratory measurements presented in Sect. 3.2 was assumed.

The KT19 collects nadir radiance with a field of view of $2^{\circ}$ while the CGR-4 measures hemispheric irradiance. The
KT19 is sensitive to wavelengths in the atmospheric window $(9.6-11.5 \mu \mathrm{m})$ and gives a measure of the surface temperature while the CGR-4 covers almost the entire terrestrial wavelength range $(4.5-42.0 \mu \mathrm{m})$ and is also sensitive to atmospheric properties (temperature and humidity profile). Therefore, a direct quantitative comparison of measurements from both instruments is not trivial. However, leads (surface temperature differences) and clouds (altitude of the emitting surface) will have similar effects on the measurements of both instruments allowing a qualitative comparison. Furthermore, the KT19 provides a higher spatial resolution due to its geometry and, therefore, serves as a reference to investigate the reconstruction of CGR-4 time series with respect to small scale fluctuations.

For the reconstruction of the time series presented in this section, the thermophile output of the CGR-4 and the sensor temperature measured by an internal thermistor were treated separately. The deconvolution was only applied to the thermophile output as the thermistor has a much slower time response and temperature changes in constant flight altitude are negligible.

\subsection{Upward irradiance over leads}

On 3 May 2012 Polar 5, operating on a north-south transect at $134.5^{\circ} \mathrm{W}$ longitude between 70.5 and $72.0^{\circ} \mathrm{N}$ latitude, crossed a cloud free area with dense sea ice. Several open leads with maximum dimensions of about $1 \mathrm{~km}$ have 
formed in the sea ice. Polar 5 flew at the low flight altitude of about $150 \mathrm{~m}$ above ground, crossing the leads. In Fig. 6, an exemplary time series of the measurements of KT19 and CGR-4 collected over leads are shown. As a comparison, the irradiance of the CGR-4 is translated into brightness temperatures using an emissivity of $\epsilon=1$. Three open leads with duration of 9,11 and $25 \mathrm{~s}(550,700$ and $1600 \mathrm{~m}$ length for ground speed of $63 \mathrm{~m} \mathrm{~s}^{-2}$ ) were observed within the $3 \mathrm{~min}$ of measurements. Mean surface temperatures of $-2.1^{\circ} \mathrm{C}$ over open water and $-7.6^{\circ} \mathrm{C}$ over the ice were observed by the KT19. The CGR-4 time series was reconstructed (red line) using a response time $\tau=3.3 \mathrm{~s}$ for the deconvolution and a cut-off frequency of $f_{\mathrm{c}}=2 \mathrm{~Hz}$. Due to the digitization of the CGR-4 AMPBOX, a moving average filter $(2 \mathrm{~s}$ window length) was applied to avoid overshootings by the deconvolution.

Comparing measured brightness temperature from CGR-4 and KT19 shows principal deviations due to the differences in the measured quantities as discussed above. The CGR-4 time series also shows the three leads but significantly lags in time due to the lagged time response of the sensor. The center time of the three leads is shifted about $2.9 \mathrm{~s}$ compared to the KT19. Similarly, the magnitude of the brightness temperatures fluctuations is affected be the slow sensor response. In the area between the first two leads, clearly identified as ice covered by the KT19, the lagged response hinders the CGR-4 signal from reaching the temperature levels measured before and afterwards above ice. In the center between the two leads, the distance of Polar 5 to the lead edges was about $250 \mathrm{~m}$. In this distance and at $150 \mathrm{~m}$ altitude the irradiance should only slightly be influenced by the leads.

These differences are partly removed in the reconstructed CGR-4 time series (red line in Fig. 6). The edges of the leads match with the KT19 data and also in the gap between the two first leads, the brightness temperatures reach the level of the sea ice area. At least over the larger third lead, the reconstructed data shortly reach an almost constant level of about $-5.2{ }^{\circ} \mathrm{C}\left(292 \mathrm{~W} \mathrm{~m}^{-2}\right)$, which can be used as a representative brightness temperature of the leads. Above the first two leads brightness temperatures increase to $-5.8^{\circ} \mathrm{C}$ $\left(290 \mathrm{~W} \mathrm{~m}^{-2}\right)$ still not reaching the value of the third lead. However, the upward terrestrial irradiance did significantly increase after reconstruction. The maximum irradiance in the two smaller leads is at least $2.5 \mathrm{~W} \mathrm{~m}^{-2}$ higher than indicated by the raw data.

\subsection{Upward irradiance over broken clouds}

On small scales clouds are highly variable especially in case of broken clouds. On 15 May 2012, Polar 5 crossed the edge of a cloud field at about $69.4^{\circ} \mathrm{N}$ and $136.6^{\circ} \mathrm{W}$ where the closed boundary layer cloud cover transformed into scatter clouds. These clouds were located over sea ice providing a high contrast between surface and cloud brightness temperatures. The corresponding measured time series of bright-

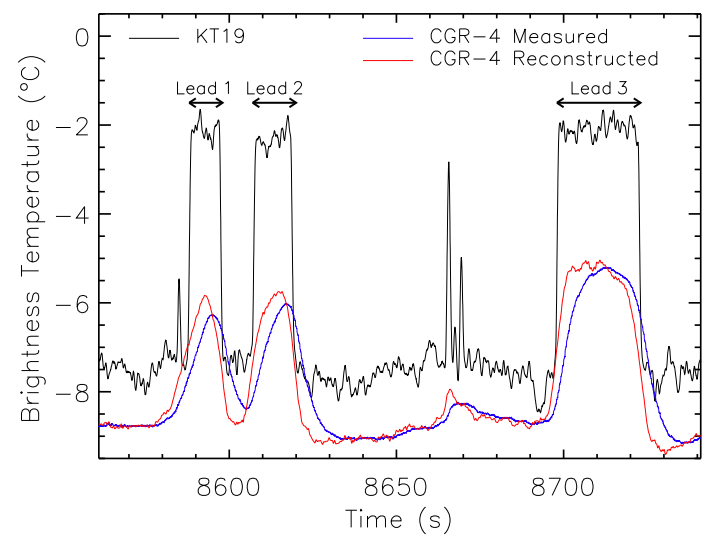

Figure 6. Brightness temperatures of upward terrestrial radiation from KT19 (black) and CGR-4 (blue) on 3 May 2012 above sea ice with leads. The red line shows the reconstructed CGR-4 data.

ness temperature of CGR-4 and KT19 are shown in Fig. 7. Within the 2 min the KT19 brightness temperature (black line) dropped by about $12 \mathrm{~K}$ when no clouds have been below the aircraft. Clouds and cloud gaps were in the range of 1-10 s flight time corresponding to sizes of $80-800 \mathrm{~m}$ at $80 \mathrm{~m} \mathrm{~s}^{-1}$ ground speed of Polar 5 .

The CGR-4 (blue line) does not show similar strong and fine scale variations as the flight altitude was about $3 \mathrm{~km}$ with cloud top altitudes at about $0.9 \mathrm{~km}$. At this distance the hemispheric integrating of the CGR-4 does not allow for the sampling of individual clouds of this size. However, by reconstructing the measured irradiance with the deconvolution method (red line) some features observed by the KT19 are also obvious in the CGR-4 time series. Even small clouds and cloud gaps such as around 2670 or 2700 s flight time become obvious in the reconstructed data.

Using the raw CGR-4 data, the period between 2660 and $2675 \mathrm{~s}$ would be identified as cloud free, and with an irradiance above sea ice of $258.5 \mathrm{~W} \mathrm{~m}^{-2}\left(13.3^{\circ} \mathrm{C}\right.$ brightness temperature) would be estimated. However, the reconstructed irradiance, resolving the small clouds, shows values of $257.0 \mathrm{~W} \mathrm{~m}^{-2}$ in the remaining cloud free areas. This is $1.5 \mathrm{~W} \mathrm{~m}^{-2}(0.4 \mathrm{~K})$ lower and illustrates that using raw CGR4 measurements can overestimate the upward irradiance of ice areas close to clouds. Similarly, the maximum brightness temperatures and irradiance above small clouds such those at 2655 or $2680 \mathrm{~s}$ is significantly underestimated by the raw pyrgeometer time series. The reconstruction results in about $0.8 \mathrm{~K}$ and $3.3 \mathrm{~W} \mathrm{~m}^{-2}$ higher values. Especially for investigations of the cloud top cooling driving cloud dynamics and entrainment at cloud top such bias might be of significance.

The reconstruction by the deconvolution method was compared to the fast approximation presented by Freese and Kottmeier (1998). Figure 8 shows CGR-4 data from Fig. 7 (red line) and results using Eq. (5) (green line). The method by Freese and Kottmeier (1998) is very sensitive to noise in 


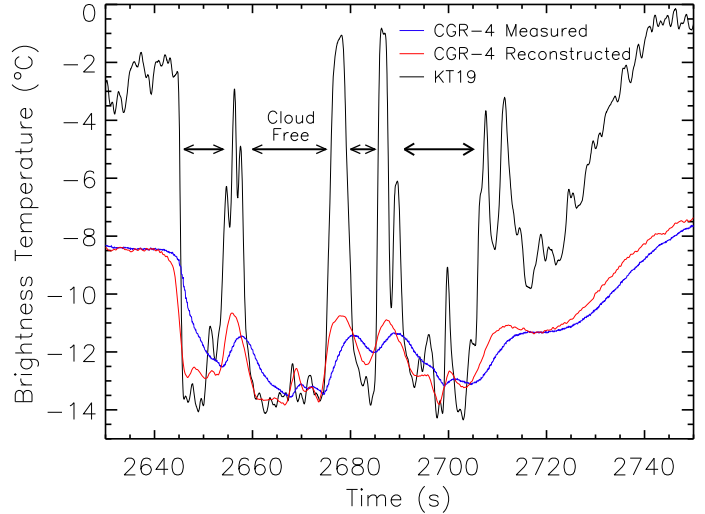

Figure 7. Brightness temperatures of upward terrestrial radiation from KT19 (black) and CGR-4 (blue) on 15 May 2015 above broken clouds. The red line shows the reconstructed CGR-4 data.

the measurements because the time derivative is calculated for each data point. We applied a moving average filter with $2.5 \mathrm{~s}$ window length to reduce noise in the CGR-4 raw data before calculating the derivative. The smoothed CGR-4 raw data are already shown in Fig. 8 (blue line). However, still the reconstruction using the approximation of Eq. (5) reveals a significantly higher noise level compared to the reconstruction using the Fourier theorem of deconvolution. A further reduction of this noise can only be achieved by smoothing the raw data a with larger window length. However, that would cause the reconstruction to fail at high frequency fluctuations. This is obvious between 2668 and $2675 \mathrm{~s}$ in Fig. 8, where the result of the approximation does not exactly follow the brightness temperatures reconstructed by the deconvolution method.

\subsection{Limitations}

The relative variations in upward terrestrial irradiance analyzed in the last sections are large. For investigations of small scale fluctuations of upward irradiance, e.g., to analyze the variability of cloud top cooling of homogeneous stratus, the resolution of the measurements has to be increased both in time and dynamic range. This is only possible if a high cutoff frequency can be selected for the inverse Fourier transformation.

For the airborne measurements presented here, $f_{\mathrm{c}}=2 \mathrm{~Hz}$ was chosen and an additional low pass filter (moving average with window length of $2 \mathrm{~s}$ ) had to be applied. Less smoothing would lead to significant overshooting by the deconvolution in the reconstructed data. This is illustrated in Fig. 9a by an exemplary $40 \mathrm{~s}$ measurement with relative low change of the terrestrial irradiance of $1 \mathrm{~W} \mathrm{~m}^{-2}$ in $20 \mathrm{~s}$ which has been reconstructed using two different configurations $\mathrm{A}$ and $\mathrm{B}$. For the data of reconstruction $\mathrm{A} f_{\mathrm{c}}=1.3 \mathrm{~Hz}$ and a moving average of $1 \mathrm{~s}$ length was used while for reconstruction $\mathrm{B}$ a moving average of $5 \mathrm{~s}$ window length was applied. For recon-

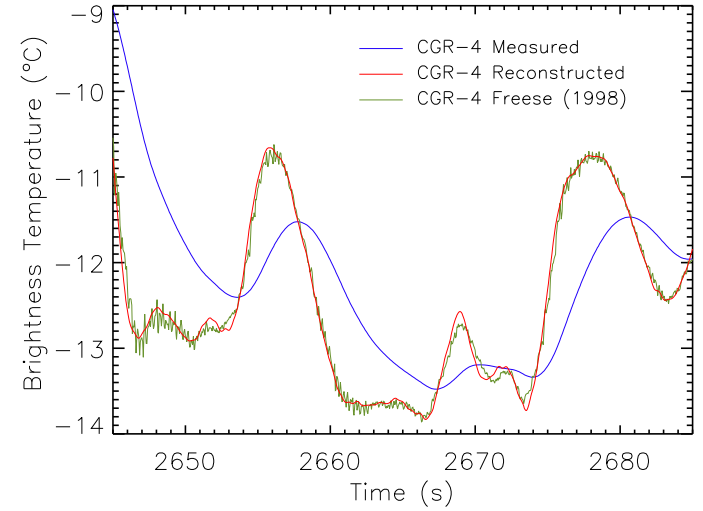

Figure 8. Comparison of reconstruction using the deconvolution method as described here and the approximation presented by Freese and Kottmeier (1998). The data are shown for the broken clouds case of 15 May 2015. The measured CGR-4 data are smoothed by a moving average filter with $2.5 \mathrm{~s}$ window length as used in the approximation.

struction A, overshootings of about $\pm 0.5 \mathrm{~W} \mathrm{~m}^{-2}$ are obvious in Fig. 9a. These overshootings correspond to steps in the raw data which can be identified by smoothing the raw data (blue line). The deconvolution transforms these steps into single positive or negative peaks.

These steps are artificial and originate from the digitalization of the amplified thermoelectric voltage by the AMPBOX amplifier. Compared to an analog signal which allows continuous values, the digital output of AMPBOX for the setup used on Polar 5 has a minimum resolution of $0.2 \mathrm{~W} \mathrm{~m}^{-2}$. To avoid overshooting due to the digitalization, the window length of the moving average filter had been increased to smooth the steps. Results using a $5 \mathrm{~s}$ window length, reconstruction B, are shown in Fig. 9a as red line. However, by the smoothing real small scale fluctuations are removed; thus, the capability of the reconstruction is reduced. The parameters of the reconstruction used for the airborne measurements represents a compromise between avoiding overshootings and resolving small fluctuations.

To show the potential of an analog amplifier, similar data (same change of terrestrial irradiance) from the CGR-4 pyrgeometer configuration used in the laboratory measurements of Sect. 3 are plotted in Fig. 9b. The maximum sampling frequency of $16 \mathrm{kHz}$ was used to demonstrate the maximal improvement. In the results using a $1 \mathrm{~s}$ averaging window length, the overshooting with about $\pm 0.1 \mathrm{~W} \mathrm{~m}^{-2}$ amplitude is significantly lower compared to the airborne measurements and can not be related to a specific pattern in the raw data. Therefore, these reconstructed fluctuation may either originate from the electronic noise or they are real. 

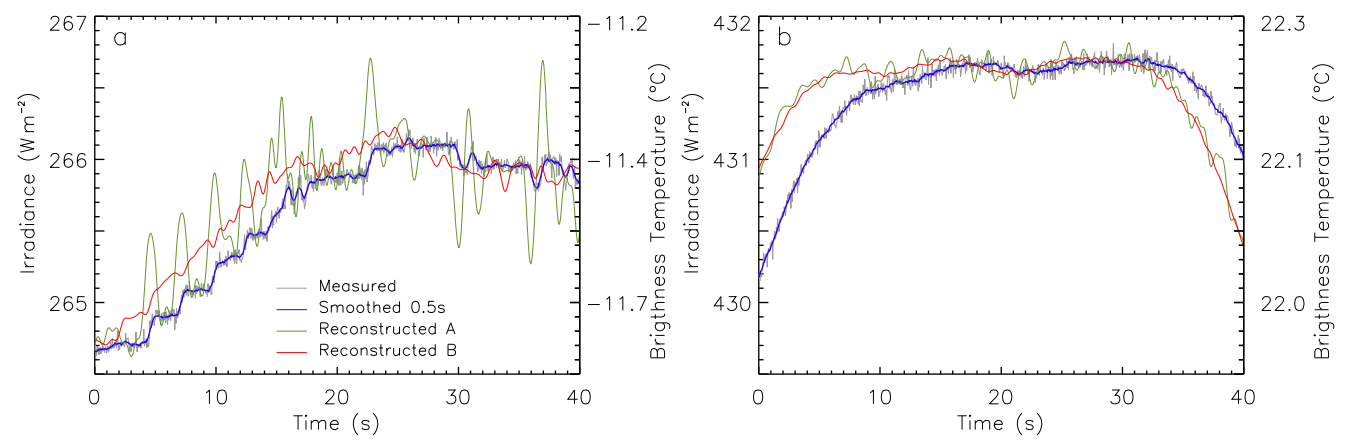

Figure 9. Comparison of the reconstruction using different low pass filter of $1 \mathrm{~s}$ window length (A) and $5 \mathrm{~s}$ window length (B). In (a) the airborne instrumentation using the digital AMPBOX are analyzed while in (b) the laboratory instrumentation with the analog signal amplifier CT 24 are shown.

\section{Conclusions}

As shown here by calculation, laboratory and field measurements the deconvolution is a powerful tool to reconstruct high-resolution time series of terrestrial irradiance measurements of pyrgeometer. By characterizing the response function of the sensor the smoothing due to the slow-response of the sensor can be removed efficiently using the convolution theorem of Fourier transform.

An empirical equation which can be used to estimate the capability of the reconstruction for measurements with $20 \mathrm{~Hz}$ recording frequency is derived. Depending on the assumed noise criteria, Eq. (12) gives the minimum amplitudes which might be reconstructed. However, comparison with laboratory measurements revealed that the estimates are optimistic. This is probably caused by the noise criteria of a signal to noise ratio of 1 which is assumed. Using a higher signal to noise ratio may provide a more conservative estimate of the amplitudes that may be resolved. Eq. (12) and the coefficients $A$ have to be considered as an upper limit of the deconvolution method.

In laboratory measurements different synthetic irradiance time series (boxcar function and periodic oscillation) have been measured by a CGR-4 pyrgeometer $(\tau=3.3 \mathrm{~s})$ and reconstructed using the deconvolution method. The results followed the theory showing that a low noise level of the measurements, achieved by a high sampling frequency, favors the reconstruction of time series contaminated by the slow response of the pyrgeometer. The higher the frequency and the lower the noise, the smaller the fluctuations which can be reconstructed because more information on the original time series is included in the measurements which can be used for reconstruction.

In case of sharp drops of the irradiance (boxcar function) the method has certain limits related to the Gibbs phenomenon. Close the edge of the shifts, ripples remain in the reconstructed time series even if a high cut off frequency is chosen. However, most applications are characterized by smooth transitions of irradiance. For idealized oscil- lations, the deconvolution method agreed with the original time series for most of the investigated sampling frequencies, oscillation frequencies and amplitudes. The normalized SD remained below $10 \%$ for oscillation frequencies of up to $0.5 \mathrm{~Hz}$ and amplitudes down to $2 \mathrm{~W} \mathrm{~m}^{-2}$. A tendency of better agreements for the oscillations with higher amplitude and high sampling frequencies was found. Oscillations with $f_{\mathrm{p}}=2 \mathrm{~Hz}$ could not be reconstructed accurately as the impact of the sensor noise is too high.

In general, the capability of the reconstruction depends on the sampling frequency and noise level of the sensor. Even time series from sensors with slow response times can be successfully reconstructed if the measured time series is provided with high temporal and dynamic resolution, high sampling frequency and low noise. In this respect, the digitalization (AD converter) of the measured signal is of high importance. To obtain accurate results from the reconstruction, a digital resolution of $14 \mathrm{bit}$ and better are beneficial. To resolve fast changes of the measured irradiance, recording frequencies of $20 \mathrm{~Hz}$ or more should be applied. A focus should be put on the noise level of the sensors. The instruments used in this study still do not apply high end AD converter. The USB-6009 by National Instruments is only at the lower end of available models with a total sampling frequency of $48 \mathrm{kHz}$ and a 14 bit resolution.

Some of these instrumental limitations have been investigated by two exemplary measurements of upward terrestrial irradiance obtained during research flights of VERDI. Over sea ice the increased surface temperature of open leads was reconstructed and compared with a KT19 infrared thermometer. The comparison illustrated that the deconvolution method is capable to correct phase shifts and reduced amplitudes of the measured fluctuations. It was shown that upward irradiance over small leads with size of about $600 \mathrm{~m}$ (10 s duration of measurement) may be underestimated by $2.5 \mathrm{~W} \mathrm{~m}^{-2}$ or more when using uncorrected measurements. Similar results were observed for the upward irradiance above broken clouds of $80-800 \mathrm{~m}$ size (1-10 s flight time) where cloud top temperatures could be improved by up to 
$1 \mathrm{~K}$. These examples revealed only a small range of applications where the reconstruction method may improve the measurement.

The reconstruction method presented here for pyrgeometer can be adapted to pyranometer and other sensors with slow response times such as contact thermometer or capacitive hygrometers. These sensors are often used in radio soundings, which also require a high temporal resolution to resolve temperature inversions or cloud layers. For example, Wang and Rossow (1995) presented a widely used method to detect clouds from radio sounding, in which one test is based on the decrease of relative humidity at cloud top. However, the humidity sensor of the often used Vaisala RS92 has a response time of $\tau=0.5-20 \mathrm{~s}$ depending on temperature. To avoid this issue, Chernykh and Eskridge (1996) developed a cloud detection algorithm based on the derivatives of temperature and humidity. However, a correct estimation of the cloud top temperature may still be biased because the RS92 temperature sensor also shows a response time of $\tau=0.4$ $2.5 \mathrm{~s}$ depending on air pressure. These values of hygrometer and thermometer are in the range of response times discussed here. Based on the theory of an exponential time response as discussed here, Miloshevich et al. (2004) developed an algorithm to reconstruct humidity soundings by successive correction the time response in each data point. They showed that especially a coarse temporal and dynamic resolution of the measurements complicate the reconstruction. Increasing the sampling frequency of these instruments and reconstructing the measurements using the deconvolution method might significantly improve the data.

Acknowledgements. We thank AWI which funded the flight operations during VERDI. Thanks also to all crew members of Polar 5 during VERDI, including scientist, engineers and pilots who made this work possible. For the support and the hospitality in Inuvik we thank the Aurora Research Center.

Edited by: C. Hoose

\section{References}

Albrecht, B. and Cox, S.: Procedures for improving pyrgeometer performance, J. Appl. Meteorol., 16, 188-197, doi:10.1175/1520-0450(1977)016<0190:PFIPP>2.0.CO;2, 1977.

Albrecht, B., Poellot, M., and Cox, S.: Pyrgeometer measurements from aircraft, Rev. Sci. Instrum., 45, 33-38, doi:10.1063/1.1686443, 1974.

Albrecht, B. A., Penc, R. S., and Schubert, W. H.: An observational study of cloud-topped mixed layers, J. Atmos. Sci., 42, 800-822, doi:10.1175/1520-0469(1985)042<0800:AOSOCT>2.0.CO;2, 1985.

Bard, E., Arnold, M., Duprat, J., Moyes, J., and Duplessy, J.C.: Reconstruction of the last deglaciation: deconvolved records of $18 \mathrm{O}$ profiles, micropaleontological variations and accelerator mass spectrometric 14C dating, Clim. Dynam., 1, 101-112, doi:10.1007/BF01054479, 1987.

Bucholtz, A., Hlavka, D. L., McGill, M. J., Schmidt, K. S., Pilewskie, P., Davis, S. M., Reid, E. A., and Walker, A. L.: Directly measured heating rates of a tropical subvisible cirrus cloud RID C-9570-2011, J. Geophys. Res., 115, D00J09, doi:10.1029/2009JD013128, 2010.

Chernykh, I. and Eskridge, R.: Determination of cloud amount and level from radiosonde soundings, $\mathrm{J}$ Appl. Meteorol., 35, 1362-1369, doi:10.1175/1520 0450(1996)035<1362:DOCAAL>2.0.CO;2, 1996.

Curry, J. A. and Herman, G. F.: Infrared radiative properties of summertime Arctic stratus clouds, J. Climate Appl. Meteor., 24, 525-538, doi:10.1175/15200450(1985)024<0525:IRPOSA>2.0.CO;2, 1985.

Duda, D. P., Stephens, G. L., and Cox, S. K.: Microphysical and radiative properties of marine stratocumulus from tethered balloon measurements, J. Appl. Meteorol., 30, 170-186, doi:10.1175/1520-0450(1991)030<0170:MARPOM>2.0.CO;2, 1991.

Foot, J.: A new prygeometer, J. Atmos. Ocean. Tech., 3, 363-370, 1986.

Freese, D. and Kottmeier, C.: Radiation exchange between stratus clouds and polar marine surfaces, Bound.-Lay. Meteorol., 87, 331-356, 1998.

Gröbner, J. and Los, A.: Laboratory calibration of pyrgeometers with known spectral responsivities, Appl. Optics, 46, 7419-7425, doi:10.1364/AO.46.007419, 2007.

Gröbner, J. and Wacker, S.: Longwave irradiance measurements using IRIS radiometers at the PMOD/WRC-IR S., Aip. Conf. Proc., 1531, 488-491, 2013

Gröbner, J., Reda, I., Wacker, S., Nyeki, S., Behrens, K., and Gorman, J.: A new absolute reference for atmospheric longwave irradiance measurements with traceability to SI units, J. Geophys. Res-Atmos., 119, 7083-7090, doi:10.1002/2014JD021630, 2014.

Klingebiel, M., de Lozar, A., Molleker, S., Weigel, R., Roth, A., Schmidt, L., Meyer, J., Ehrlich, A., Neuber, R., Wendisch, M., and Borrmann, S.: Arctic low-level boundary layer clouds: in situ measurements and simulations of mono- and bimodal supercooled droplet size distributions at the top layer of liquid phase clouds, Atmos. Chem. Phys., 15, 617-631, doi:10.5194/acp-15617-2015, 2015.

Lüpkes, C., Vihma, T., Birnbaum, G., and Wacker, U.: Influence of leads in sea ice on the temperature of the atmospheric boundary layer during polar night, Geophys. Res. Lett., 35, L03805, doi:10.1029/2007GL032461, 2008.

Meloni, D., Di Biagio, C., di Sarra, A., Monteleone, F., Pace, G., and Sferlazzo, D. M.: Accounting for the solar radiation influence on downward longwave irradiance measurements by pyrgeometers, J. Atmos. Ocean. Tech., 29, 1629-1643, doi:10.1175/JTECH-D-11-00216.1, 2012.

Miloshevich, L., Paukkunen, A., Vomel, H., and Oltmans, S.: Development and validation of a time-lag correction for Vaisala radiosonde humidity measurements, J. Atmos. Ocean. Tech., 21, 1305-1327, doi: 10.1175/15200426(2004)021<1305:DAVOAT>2.0.CO;2, 2004. 
Philipona, R., Fröhlich, and Betz, C.: Characterisation of pyrgeometers and the accuracy of atmospheric long-wave radiation measurements, Appl. Optics, 34, 1598-1605, 1995.

Philipona, R., Dutton, E., Stoffel, T., Michalsky, J., Reda, I., Stifter, A., Wendling, P., Wood, N., Clough, S., Mlawer, E., Anderson, G., Revercomb, H., and Shippert, T.: Atmospheric longwave irradiance uncertainty: Pyrgeometers compared to an absolute sky-scanning radiometer, atmospheric emitted radiance interferometer and radiative transfer model calculations, J. Geophys. Res., 106, 28129-28141, 2001.

Ruffieux, D., Persson, P. O. G., Fairall, C. W., and Wolfe, D. E.: Ice pack and lead surface-energy budgets during LEADEX-1992, J. Geophys. Res., 100, 4593-4612, doi:10.1029/94JC02485, 1995.

Saunders, R. W., Brogniez, G., Buriez, J. C., Meerkötter, R., and Wendling, P.: A comparison of measured and modeled broadband fluxes from aircraft data during the ICE' 89 field experiment, J. Atmos. Ocean. Tech., 9, 391-406, 1992.
Schäfer, M., Bierwirth, E., Ehrlich, A., Jäkel, E., and Wendisch, M.: Airborne observations and simulations of three-dimensional radiative interactions between Arctic boundary layer clouds and ice floes, Atmos. Chem. Phys., 15, 8147-8163, doi:10.5194/acp15-8147-2015, 2015.

Wang, J. and Rossow, W.: Determination of cloud vertical structure from upper-air observations, J. Appl. Meteorol., 34, 2243-2258, 1995.

Wendisch, M. and Brenguier, J.-L.: Airborne Measurements for Environmental Research - Methods and Instruments, Wiley-VCH Verlag GmbH and Co. KGaA, Weinheim, Germany, ISBN9783-527-40996-9, 641 pp., 2013.

Wilson, J. D., Massman, W. J., and Swaters, G. E.: Dynamic response of the thermometric net radiometer, Agr. Forest Meteorol., 149, 1358-1364, doi:10.1016/j.agrformet.2009.03.007, 2009.

Wood, R.: Stratocumulus clouds, Mon. Weather Rev., 140, 23732423, 2012. 